\title{
Large micro-mirror arrays: key components in future space instruments for Universe and Earth Observation
}

\author{
Frederic Zamkotsian ${ }^{1, a}$, Patrick Lanzoni ${ }^{1}$, Michael Canonica ${ }^{2}$, Wilfried Noell ${ }^{2}$, Sebastien Lani ${ }^{3}$ \\ ${ }^{1}$ Laboratoire d'Astrophysique de Marseille - CNRS, 13388 Marseille Cedex 13, France \\ ${ }^{2}$ Ecole Polytechnique Fédérale de Lausanne, CH-2002 Neuchâtel, Switzerland \\ ${ }^{3}$ CSEM, CH-2002 Neuchâtel, Switzerland
}

\begin{abstract}
In future space missions for Universe and Earth Observation, scientific return could be optimized using MOEMS devices. Micro-mirror arrays are used for designing new generation of instruments, multi-object spectrographs in Universe Observation and programmable wide field spectrographs in Earth Observation. Mock-ups have been designed and built for both applications and they show very promising results.
\end{abstract}

\section{Introduction}

Micro-Opto-Electro-Mechanical Systems (MOEMS) will be key components in future generation of space instruments. In Earth Observation, Universe Observation and Planet Exploration, scientific return of the instruments must be optimized in future missions. MOEMS devices are based on the mature microelectronics technology and in addition to their compactness, scalability, and specific task customization, they could generate new functions not available with current technologies.

French and European space agencies, the Centre National d'Etudes Spatiales (CNES) and the European Space Agency (ESA) have initiated several studies with LAM for listing the new functions associated with several types of MEMS, and developing new ideas of instruments. [1]

\section{Programmable Multi-Object Spectroscopy for Universe Observation}

\subsection{Multi-Object Spectroscopy}

Multi-Object Spectrographs (MOS) are the major instruments for studying primary galaxies and remote and faint objects. Current object selection systems are limited and/or difficult to implement in next generation MOS for space and ground-based telescopes. A promising solution is the use of MOEMS devices such as micromirror arrays which allow the remote control of the multi-slit configuration in real time. The programmable slit mask is placed at the focal plane of the telescope for astronomical object selection at the entrance of the spectrograph (Fig. 1).

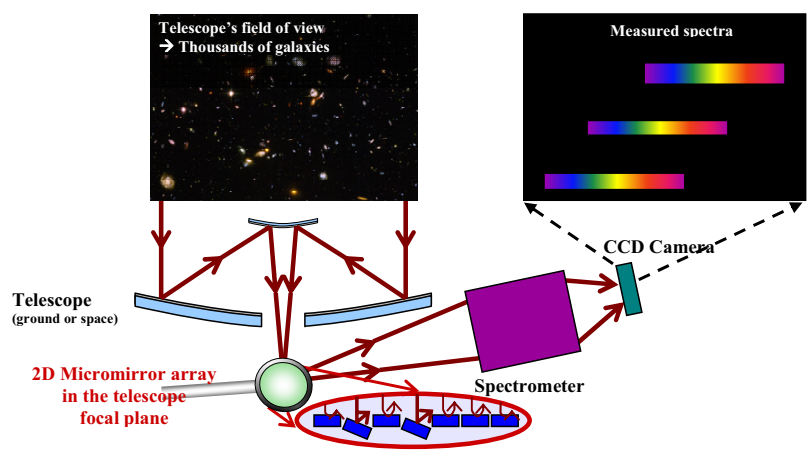

Figure 1. Multi-object spectroscopy principle.

\subsection{MIRA}

Collaboration within Laboratoire d'Astrophysique de Marseille (LAM), Ecole Polytechnique Fédérale de Lausanne (EPFL) and Centre Suisse d'Electronique et de Microtechnique (CSEM) has for purpose to develop a European programmable micromirror array (MMA), called MIRA, for generating reflective slit masks in future MOS. The requirements were determined from previous simulation results and measurements. It has to achieve a high optical contrast of 1000:1 (goal: 3000:1), a fill factor of more than $90 \%$ and a mechanical tilt angle greater than $20^{\circ}$. Furthermore, the mirror surface must remain flat in operation throughout a large temperature range and it has to work at cryogenic temperature.

MMA with $100 \times 200 \mu \mathrm{m}^{2}$ single-crystal silicon micromirrors were successfully designed, fabricated and tested (Fig. 2). Arrays are composed of 2048 micromirrors $(32 \times 64)$ with a peak-to-valley deformation less than $10 \mathrm{~nm}$, a tilt angle of $24^{\circ}$ for an actuation voltage of $130 \mathrm{~V}$. [2]

\footnotetext{
${ }^{\mathrm{a}}$ Corresponding author: frederic.zamkotsian@lam.fr
} 
The micromirrors were actuated successfully before, during and after cryogenic cooling, down to $162 \mathrm{~K}$. The micromirror surface deformation was measured at cryo and is below $30 \mathrm{~nm}$ peak-to-valley.

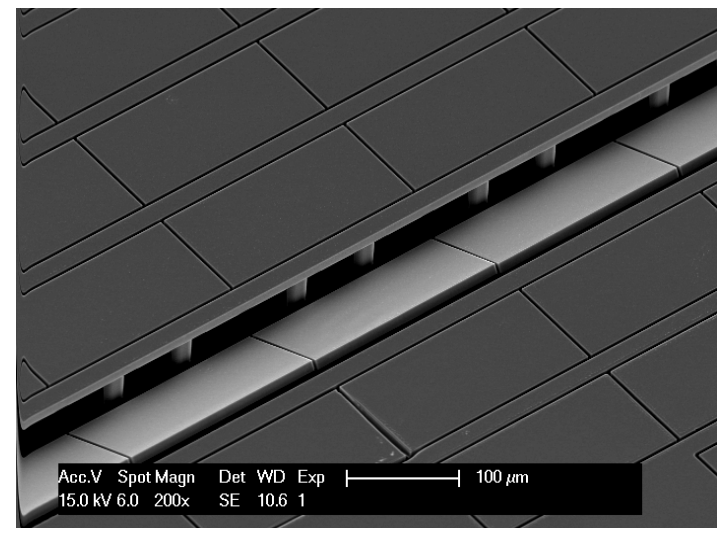

Figure 2. MIRA, a micro-mirror array composed of 2048 mirrors, with an individual size of $100 \times 200 \mu \mathrm{m}^{2}$.

\subsection{MOS demonstrators: BATMAN and ROBIN}

We are developing a Digital Micromirror Device (DMD) - based spectrograph demonstrator called BATMAN. We want to access the largest FOV with the highest contrast. The selected component is a DMD chip from Texas Instruments in $2048 \times 1080$ mirrors format, with a pitch of $13.68 \mu \mathrm{m}$. Our optical design is an all-reflective spectrograph design with $\mathrm{F} / 4$ on the DMD component.

This demonstrator permits the study of key parameters such as throughput, contrast and ability to remove unwanted sources in the FOV (background, spoiler sources), PSF effect, new observational modes. This study will be conducted in the visible with possible extension in the IR.

The demonstrator on the sky is then of prime importance for characterizing the actual performance of this new family of instruments, as well as investigating the operational procedures on astronomical objects. BATMAN will be placed on the Nasmyth focus of Telescopio Nazionale Galileo (TNG) during next year.

Before developing BATMAN, we have built a demonstrator named ROBIN, for characterizing the actual performance of this new family of instruments, as well as investigating the operational procedures on astronomical objects. The design of the demonstrator is identical to the instrument design for being fully representative, with a global reduced size, on mirrors as well as on the grating. The general mechanical design of ROBIN consists of a main optical bench supporting 2 arms: a spectrograph arm and an imaging arm. The detectors are located on both sides of the bench. Optomechanical design is shown in Fig. 3.

ROBIN has been integrated and aligned (Fig. 4). The optical beam is entering from the top center; the DMD is located at the bottom center and both arms are fed, on the right hand side is the imaging arm and on the left hand side is the spectroscopic arm.

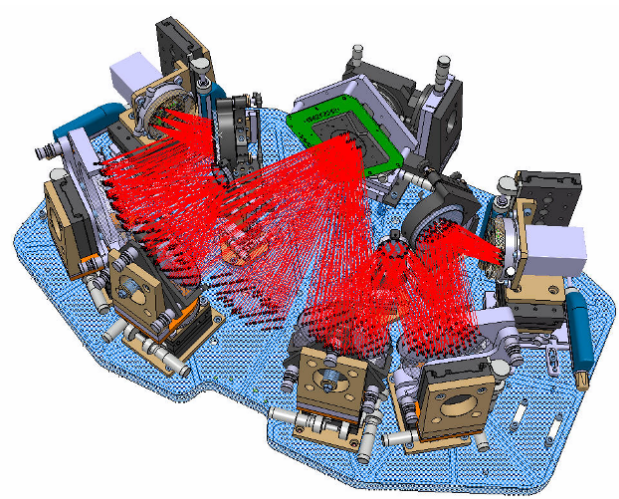

Figure 3. 3D general design view of ROBIN (in red, beam propagation in the demonstrator);

Both arms are fully identical except the convex mirror being replaced by the convex grating in the spectroscopic arm. Images and spectra are recorded by two CCD cameras located on both sides (left and right).

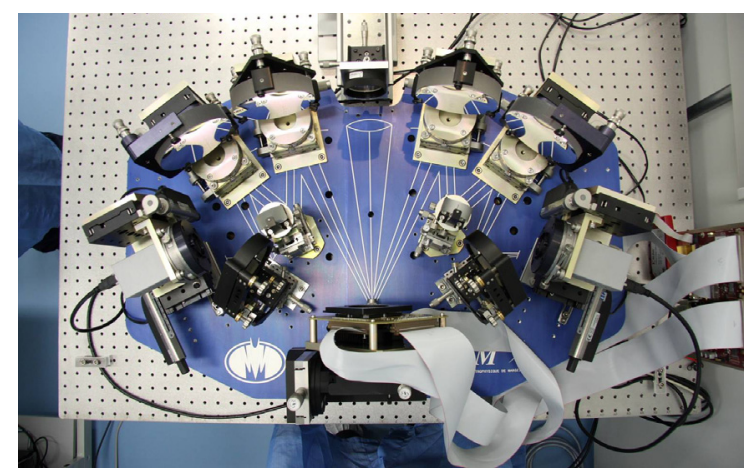

Figure 4. Integrated ROBIN picture.

First images and spectra have been obtained and measured. In the imaging arm, typical slit mask patterns are recorded (Fig. 5); the optical quality is good enough for imaging each individual micromirror.

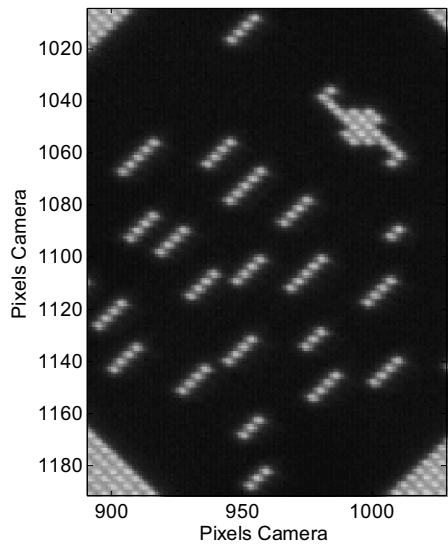

Figure 5. Image of a typical slit mask in the imaging channel.

In the spectroscopic arm, typical spot diameters are within 1.5 detector pixels, and spectra generated by one micro-mirror slits are displayed with this optical quality over the whole visible wavelength range (Fig. 6). 


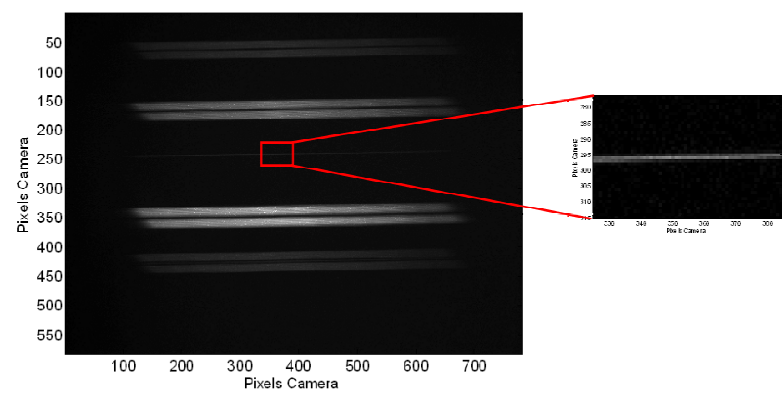

Figure 6. Spectra in the spectral channel, including a single micromirror slit (close view of the spectrum generated by the one-micromirror slit).

We have tested the instrument abilities in terms of variable spatial bin and variable spectral resolution, and any combination of the above modes over the whole FOV; in particular, MOS and IFU-like (scanning slit) modes have been studied, with any slit mask configurations (any shape, including long slit) as well as real time reconfiguration.

These performances demonstrate the ability of such MOEMS device to work as objects selector in future generation of MOS instruments both in ground-based and space telescopes. In order to fill large focal planes (mosaicing of several chips), we are currently developing large micromirror arrays integrated with their electronics.

\section{Programmable wide-field spectrograph for Earth Observation}

In Earth Observation, we propose an innovative reconfigurable instrument, a programmable wide-field spectrograph where both the FOV and the spectrum could be tailored thanks to a 2D micromirror array (MMA).

For a linear 1D field of view (FOV), the principle is to use a MMA to select the wavelengths by acting on intensity (Fig. 7).

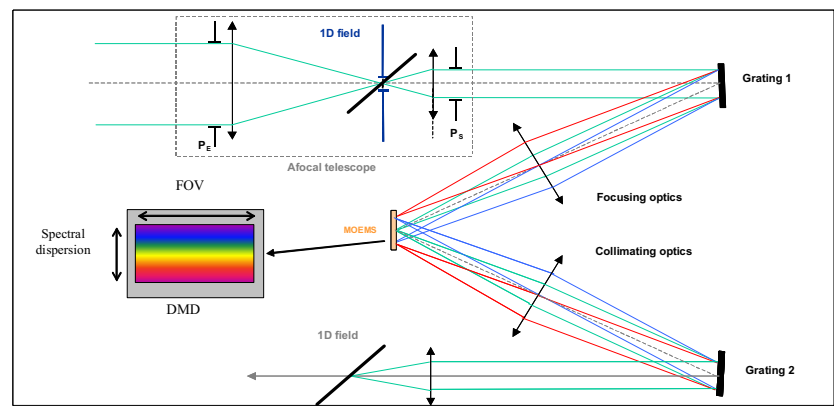

Figure 7. Programmable wide field spectrograph principle.

This component is placed in the focal plane of a first grating. On the MMA surface, the spatial dimension is along one side of the device and for each spatial point, its spectrum is displayed along the perpendicular direction: each spatial and spectral feature of the 1D FOV is then fully adjustable dynamically. A second stage with an identical grating recomposes the beam after wavelengths selection, leading to an output tailored 1D image.

A mock-up has been designed, fabricated and tested (Fig. 8). The micromirror array is the largest DMD in 2048 x 1080 mirrors format. A synthetic linear FOV is generated and typical images have been recorded at the output focal plane of the instrument. By tailoring the DMD, we could modify successfully each pixel of the input image: for example, it is possible to remove bright objects or, for each spatial pixel, modify the spectral signature [3].

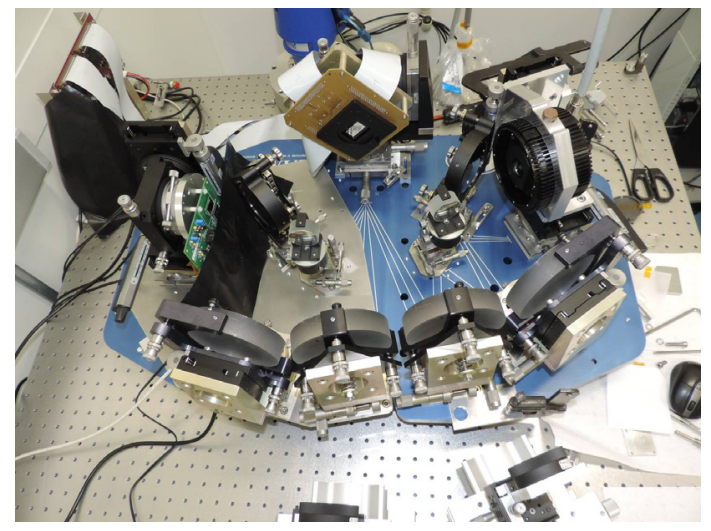

Figure 8. Mock-up of the programmable wide field spectrograph.

\section{Conclusion}

In Earth Observation, Universe Observation and Planet Exploration, scientific return of the instruments must be optimized in future missions. Micro-Opto-ElectroMechanical Systems (MOEMS) could be key components in future generation of space instruments. We are developing a European micro-mirror array called MIRA and able to operate at cryogenic temperatures.

We have also designed and tested successfully new concepts for Multi-Object Spectroscopy as well as for programmable wide field spectrographs.

\section{References}

1. F. Zamkotsian, A. Liotard, P. Lanzoni, T. Viard, "Optical MEMS in space instruments for Earth Observation and Astronomy", Proceedings of the SPIE conference on MOEMS 2013, Proc. SPIE 8616, San Francisco, USA, (2013)

2. M. Canonica, F. Zamkotsian, P. Lanzoni, W. Noell, N. de Rooij, "The two-dimensional array of 2048 tilting micromirrors for astronomical spectroscopy," Journal of Micromechanics and Microengineering, 23 055009, (2013)

3. F. Zamkotsian, P. Lanzoni, A. Liotard, T. Viard, V. Costes, P.-J. Hebert, "DMD-based programmable wide field spectrograph for Earth Observation", Proceedings of the SPIE conference on MOEMS 2015, Proc. SPIE 9376, San Francisco, USA, (2015) 